\title{
Professional Role Performance in Journalism
}

\author{
Interview with Claudia Mellado \\ Pontificia Universidad Católica de Valparaíso, Chile
}

You have just published your new book, entitled Beyond Journalistic Norms. Is hybridization of journalistic cultures an irreversible process?

Hybridity is inherent to journalism. If you think about it, journalists constantly need to adjust and perform multiple roles. For example, with the COVID19 pandemic, the world has seen that news professionals have an extraordinary capacity to adapt their roles and practice to changing circumstances, so the idea of conceiving journalistic cultures as static and monolithic oversimplifies the complex reality that our profession is going through. Journalistic roles are situational, dynamic, and fluid, and cannot be considered discrete categories. Even if we as journalists adhere to specific journalistic norms, it is completely understandable that context-specific roles will guide their everyday work.

In our new book, Beyond Journalistic Norms (Routledge, 2021), we go in-depth into that discussion. We start from the position that journalistic practice transcends normative expectations, challenging pre-established assumptions about a "dominant type" of journalism that prevails in different political, geographic, and cultural contexts. Our studies suggest that there is no single dominant journalistic model across countries and that journalistic cultures are based on a combination of role performances.

Models of journalistic professionalism coming from the Western world have significantly impacted our mindsets when thinking about this topic. That is why journalism students around the world learn that facts must always be separated from opinions, that hard news are serious while soft news are not, and that journalists should always act as watchdogs but never perform loyalism because both are not compatible with each other. These norms are indeed pedagogical for constructing discourses of occupational legitimacy. Still, we shouldn't think about journalism in the abstract because, in practice, things do not work in that way, so those binary expectations do not help us understand how journalism and the media actually work. Because of the same, it is also dangerous to make 
absolute claims about the relationship between the practice of our profession and concepts that are quite culturally bound, such as democracy, objectivity, autonomy, among others.

From the perspective of role performance, roles should not be taken as uniform, whole constructs but instead as permeable practices. Professional roles are intermediary translation devices in the process of practicing the profession. Because of that, I strongly believe that identifying the practical co-occurrences of different roles is key to better understand the multiple layers of hybridization across and within journalistic cultures. In our studies we argue that roles interact with one another, creating potentially intermediate roles. We have already found strong evidence of a significant co-occurrence of professional roles used simultaneously by journalists around the world to inform the public. For example, about the audience approach domain, we have found a strong tendency to blend the performance of the interventionist role with the infotainment role in the print media of most countries. In several countries, we have also seen the co-occurrence of the watchdog and civic roles, of the civic and the loyal role, and the infotainment and civic roles, and even sometimes, of all of them together.

Journalists work for different media platforms, create stories from different beats, and write for many different audiences simultaneously. Their work can also change dramatically from one day to the next, and they must occasionally negotiate their ideals with situations that require specific role performances. This means that a single news story must meet different requirements associated with the merging of diverse roles. For example, they can be critical of the president but praise the leader of the opposition in a country; or inform citizens of their duties and rights in an entertaining way. We should not be that surprised that this happens anyways. The co-existence of roles is inherent to any given occupation because people need to respond to ongoing demands in complex societies like ours.

How do you see the future of international studies of journalistic cultures and professionalization that adopt a role performance approach? What kind of research is now needed the most?

Our Journalistic Role Performance (JRP) project was pioneering in that it was the first one that empirically unfolded the different layers of journalistic cultures through the study of both ideals and professional practices around the world. Nevertheless, science is always a work in progress. There are so many things to learn, discover, improve, change, and develop.

One of the major limitations of the first wave of our JRP project was that it focused on analyzing a single media platform: print media. The project demonstrated that the analysis of national print media systems was a necessary 
and useful stage for analyzing professional roles in different contexts. However, we know that the practice of journalism is much broader, including multimedia and audiovisual dynamics, and social media and interactive elements that are nonexistent in print. Another important limitation was the inclusion of news from the National Desk only, ignoring how professional roles may play out differently in other topics. In acknowledging our limitations, the new wave of our project (2019-2021) analyzes professional roles and journalistic performance on different traditional media platforms, and in different news topics/ beats. Nevertheless, we have not included social media platforms in our analysis, so definitively that is a much-needed contribution that future research must accomplish, especially in terms of the developments of news measures that can better address the specific media logics of social media spaces.

Role performance research also poses new challenges that can serve as a roadmap for future scholarship considering the complexity of the factors that influence journalistic cultures. For example, future studies should be able to approach this topic using different methodologies. On the one hand, qualitative studies have a lot to contribute in this regard. On the other hand, the automatization of some measures and the use of machine learning techniques can also bring new possibilities to this area of study.

About the performance of journalism itself, future research could make a tremendous contribution thinking not only about how journalists perceive and practice their profession but also about the impact of the audience on current journalistic ideals and practices. Future work might also consider studying more in-depth the blending of different roles when journalists cover specific events that transcend national boundaries, such as COVID19.

\section{Are media systems and journalistic cultures too complex to cluster?}

Both media systems and journalistic cultures are complex indeed. From the perspective of role performance research, I can say that the fluid and contextual practice of journalism is much more difficult to fit in existing typologies than specific media system categories. Media systems are not static either and also change and evolve, but the pace of their change is different.

If we look at the interplay between both media systems and journalistic cultures, we can then see to what extent media system categories hold true in shaping reporting practices and narratives. So far, our studies have shown that media systems theory is especially useful for predicting the performance of roles related to the public sphere. About these roles, our results reveal that news media are shaped by society and politics much more than news media actively shape society and politics. Nevertheless, we have found that media system models lack explanatory power for other roles. 
Should we then forget about models of media systems?

No, of course not. Media systems' models identify crucial political, economic, social, and cultural characteristics that determine the context in which the media operate, so they are beneficial for understanding how journalism work at the structural level, as well as the relationship between macro-level factors and professional roles associated with the public sphere as I mentioned in the previous question. What is necessary, though, is more theory on media systems outside the Western world. Specifically, media systems scholars must develop new dimensions that allow for the inclusion of realities of countries from Asia, Africa, Eastern Europe, and Latin America, which have experienced different levels of conceptual dependency in this area of study.

How would you describe the state-of-the-art media and journalism studies in Latin America?

Media studies in Latin America developed significantly more than journalism studies, especially because of the interests of most communication scholars in the region since the field started to grow. But things have changed lately. Until two decades ago, studies on Latin American journalism were marginal and mostly related to working conditions. There was no real interest in theorizing about the profession or empirically addressing global questions that could help understand journalistic ideologies and practices compared to other cultures. Journalism was not considered as a proper field but more as an appendix of communication studies. Thank God globalization has helped connect Latin American scholars with the rest of the world and professionalize journalism studies in the region. Of course, some countries show much more representation than others if we look at their publications and what we know about journalism within Latin America, but the field has started to flourish.

Are Latin American researchers sufficiently represented in the global context?

My answer is no, we are not visible enough. With some exceptions, more Latin Americans that stand out in the international or global context are the ones that moved to the U.S. or Western countries a long time ago. In the meantime, there are hundreds of incredible scholars in Latin America that are not visible in a mainstream context, although their work largely contributes to our understanding of the profession. I have seen the establishment of some politically correct current discourse about more equality in terms of scholars' representations in the academic field. That is quite positive, but I would like to see a bit 
more than only a discourse. On the other hand, I would also like to see more proactivity from the part of some Latin American colleagues as well.

Besides having a very marginal representation in the field, we are always in disadvantage. We always need to convince reviewers and editors every time we want to publish our work on why studying a Latin American country or the region is relevant for academic purposes. I cannot imagine asking that question to a Western European scholar or U.S. researchers. Language is another barrier, but I believe that the main issue is that we are still not accepted as a "legitimate other" in the field. We still need to justify our existence. I strongly believe that research creates significant knowledge when questioning the familiar and the known, opening the scope to the unexplored. And there is a lot to do in this regard.

Claudia Mellado was interviewed by Michał Kuś in November 2020.

Claudia Mellado is Professor of Journalism in the School of Journalism at Pontificia Universidad Católica de Valparaíso (PUCV), Chile. Before joining PUCV she taught at the University of Santiago and the University of Concepcion in the same country. She received her doctoral degree in Communication from the Pontifical University of Salamanca, Spain in 2004. During 2007-2008, she did her postdoctoral work at the School of Journalism, Indiana University. Her research focuses on the study of journalism cultures, journalistic role performance, and comparative studies. She is the principal investigator of the JRP Project (www. journalisticperformance.org). Her work has been extensively published in journals such as Journal of Communication, International Journal of Press/Politics, Journalism, JMCQ, Journalism Studies, Journalism Practice, Communication Theory, and Digital Journalism. Her last edited books are Journalistic Role Performance: Concept, Contexts, and Methods (Routledge, 2017), and Beyond Journalistic Norms. Role Performance and News in Comparative Perspective (Routledge, 2021). 